\title{
Dynamic changes in chest CT findings of patients with coronavirus disease 2019 (COVID-19) in different disease stages: a multicenter study
}

Yilong Huang ${ }^{1 \#}$, Zhipeng $\mathrm{Li}^{2 \#}$, Honglei Guo ${ }^{1 \#}$, Dan $\mathrm{Han}^{1}$, Feng Yuan ${ }^{1}$, Ying $\mathrm{Xie}^{3}$, Zhi Li ${ }^{4}$, Jing Zhang ${ }^{5}$, Peng Wang ${ }^{6}$, Yan Yang ${ }^{7}$, Yudan $\mathrm{Li}^{1}$, Jiyao Ma ${ }^{1}$, Chunzhang $\mathrm{Hu}^{1}$, Haolei Wang ${ }^{1}$, Wei Zhao ${ }^{1}$, Wenfang Yi ${ }^{1}$, Yan Peng ${ }^{8}$, Yuanming Jiang ${ }^{1}$, Zhanfeng Li $^{9}$, Duiming Yang ${ }^{10}$, Kaiming Wu ${ }^{11}$, Yuchang Li ${ }^{12}$, Zaihui Feng ${ }^{13}$, Chaohai Liu ${ }^{14}$, Guangkun Zhu ${ }^{15}$, Yongyun $\mathrm{Han}^{16}$, Shaofei $\mathrm{He}^{17}$, Bo He ${ }^{1}$, Zhenguang Zhang ${ }^{1}$, Jiangyuan $\mathrm{Pi}^{18}$

${ }^{1}$ Medical Imaging Department, First Affiliated Hospital of Kunming Medical University, Kunming, China; ${ }^{2}$ Medical Imaging Department, Yunnan Provincial Infectious Disease Hospital (Yunnan AIDS Care Center), Kunming, China; ${ }^{3}$ Department of Radiology, Calmette Hospital \& The First Hospital of Kunming, Kunming, China; ${ }^{4}$ Department of Radiology, The First People's Hospital of Yunnan Province, Kunming, China; ${ }^{5} \mathrm{CT}$ Room, People's Hospital of Yuxi City, Yuxi, China; ${ }^{6}$ Radiology Department, The Second People's Hospital of Yunnan Province, Kunming, China; ${ }^{7}$ Radiology Department, The Third People's Hospital of Yunnan Province, Kunming, China; ${ }^{8}$ Department of Radiology, the First People's Hospital of Zhaotong, Zhaotong, China; ' Department of Radiology, The First People's Hospital of Jinghong City, Xishuangbanna Prefecture, Jinghong, China; ${ }^{10}$ Medical Imaging Department, The Second People's Hospital of Baoshan City, Baoshan, China; ${ }^{11}$ Department of Radiology, Honghe County People's Hospital, Honghe Prefecture, Honghe, China; ${ }^{12}$ Department of Radiology, Midu People's Hospital, Midu, China; ${ }^{13}$ Honghe Autonomous Prefecture Third Hospital, Honghe, China; ${ }^{14}$ Medical Imaging Department, The First People's Hospital of Xichou, Xichou, China; ${ }^{15}$ Medical Imaging Department, People's Hospital of Yuanyang County, Honghe Prefecture, Honghe, China; ${ }^{16}$ Department of Medical Imaging, Longling County People's Hospital, Longling, China; ${ }^{17}$ Medical Imaging Department, The People's Hospital of Mengzi, Mengzi, China; ${ }^{18}$ Kunming Medical University, Kunming, China

Contributions: (I) Conception and design: Y Huang, Z Li, H Guo, B He; (II) Administrative support: D Han, F Yuan, Y Xie, Z Li, J Zhang, P Wang, Y Yang, B He, Z Zhang, J Pi; (III) Provision of study materials or patients: Y Xie, Z Li, J Zhang, P Wang, Y Yang, Y Peng, Z Li, D Yang, K Wu, Y Li, Z Feng, C Liu, G Zhu, Y Han, S He; (V); (IV) Collection and assembly of data: Y Li, J Ma, C Hu, H Wang, Y Jiang; (V) Data analysis and interpretation: Y Huang, Z Li, H Guo, W Zhao, W Yi, B He; (VI) Manuscript writing: All authors; (VII) Final approval of manuscript: All authors.

"These authors contributed equally to this work.

Correspondence to: Bo He. Medical Imaging Department, First Affiliated Hospital of Kunming Medical University, Kunming Medical University, Kunming, China. Email: hebo_ydyy@qq.com; Zhenguang Zhang. Medical Imaging Department, First Affiliated Hospital of Kunming Medical University, Kunming Medical University, Kunming, China. Email: 178546941@qq.com; Jiangyuan Pi. Kunming Medical University, Kunming, China. Email: 493335833@qq.com.

Background: To investigate the dynamic changes in high-resolution computed tomography (HRCT) findings of coronavirus disease 2019 (COVID-19) patients with different severities in different disease stages. Methods: We retrospectively collected the clinical and imaging data of 96 patients in Yunnan Province, China, who were diagnosed with COVID-19 between January 22 and March 15, 2020. Based on disease severity, the COVID-19 patients were classified into four types: mild ( $n=15)$, moderate $(n=59)$, severe $(n=19)$, and critical $(n=3)$. Based on hospital stay and number of computed tomography (CT) scans, the clinical/ disease course was divided into four stages, including stage 1 (days 0-4), stage 2 (days 5-9), stage 3 (days 10-14), and stage 4 (days 15-19). The HRCT findings, CT value, and lesion volume were analyzed for each stage and compared among the four stages of COVID-19 patients.

Results: CT findings were negative over the four stages for all mild COVID-19 patients. More lesions were found in the peripheral lung fields than in peripheral + central fields $(\mathrm{P}<0.05)$, and the number of negative patients in stage 4 were more than those in stages $1-3(\mathrm{P}<0.05)$. The left and right lower lobe were the most frequently affected lobes $(\mathrm{P}<0.05)$. In moderate patients, round ground glass opacities $(\mathrm{GGOs})$ decreased from stage 1 to stage 4; partial consolidation peaked in stage 2 and then decreased in stages 3-4; 
fibrous stripes and subpleural lines increased from stage 1 and peaked in stage 4. Partial consolidation and consolidation were more common in severe patients than in moderate patients over the disease course $(\mathrm{P}<0.05)$. Critical patients showed significant partial consolidation and consolidation; The CT value, lesion volume and lesion volume percentage significantly decreased from stages $1-2$ to stage 4 (all $\mathrm{P}<0.05$ ).

Conclusions: The dynamic changes in lung HRCT images are clinically related to the disease course of COVID-19.

Keywords: Coronavirus disease 2019 (COVID-19); pneumonia; computed tomography (CT); X-ray computed tomography (X-ray CT)

Submitted Nov 19, 2020. Accepted for publication Jan 18, 2021.

doi: 10.21037/apm-20-2484

View this article at: http://dx.doi.org/10.21037/apm-20-2484

\section{Introduction}

Coronavirus disease 2019 (COVID-19) is a highly infectious disease caused by severe acute respiratory syndrome coronavirus 2 (SARS-CoV-2) and has a long incubation period. It mainly causes lung infection (1). COVID-19 is spreading at an alarming rate; as of March 20, it has spread to 77 countries worldwide, with 268,404 confirmed patients and 11,215 deaths. COVID-19 has become a global health crisis. With the gradual deepening of the understanding of COVID-19, Chinese authorities have established expert consensus, guidelines, and standards in order to prevent further spread and improve diagnosis and treatment (2). Computed tomography (CT) plays a key role in COVID-19 diagnosis and treatment (3). Studies have shown that some COVID-19 patients present little or no clinical symptoms and display negative CT findings during hospitalization $(4,5)$, some patients develop difficulty breathing and/or hypoxemia within one week of onset, and some severe patients develop acute respiratory distress syndrome, septic shock, refractory metabolic acidosis, coagulation disorders, and multiple organ failure $(6,7)$. In addition, studies have shown that the lung lesions in COVID-19 patients evolve over the disease course, as evidenced by CT findings. In the early stage, CT shows round ground glass opacities (GGOs) and partial consolidation. As the disease progresses, CT shows bilateral diffuse GGOs and consolidation (8). These data indicate that CT findings are diverse and complex over the disease course in COVID-19 patients with different severities. Currently, the "gold standard" is real-time reverse transcriptase polymerase chain reaction (RT-PCR) amplification of the viral DNA for diagnosis of COVID-19. However, CT is of great significance for monitoring the development of pneumonia caused by COVID-19.
This study was designed to investigate the dynamic changes in CT findings of COVID-19 patients with different severities in different disease stages.

We present the following article in accordance with the MDAR checklist (available at http://dx.doi.org/10.21037/ apm-20-2484).

\section{Methods}

\section{Patients}

The study was conducted in accordance with the Declaration of Helsinki (as revised in 2013). The retrospective study was approved by the institutional committee of the First Affiliated Hospital of Kunming Medical University. Informed consent was waived because the study was retrospective in design. In this multicenter study, we retrospectively analyzed the clinical data of patients with suspected COVID-19 who underwent chest CT due to fever, fatigue, or respiratory symptoms between January 22 and March 15, 2020. The diagnostic criteria for COVID-19 are as follows: (I) two positive SARS$\mathrm{CoV}$-2 tests by reverse transcription polymerase chain reaction (RT-PCR) and (II) negative nucleic acid tests for other known respiratory viruses. The selection process for COVID-19 patients is shown in Figure 1. An overall total of 96 COVID-19 patients were included in this study. All patients were admitted within 4 days of disease onset. The information collected for each patient included age, sex, history of exposure, clinical symptoms (fever, cough, fatigue, difficulty breathing, sore throat, runny nose, sputum, headache, muscle aches, chest tightness, chest pain, nausea and vomiting, diarrhea, or no symptoms), underlying diseases (hypertension, diabetes, coronary heart disease, 


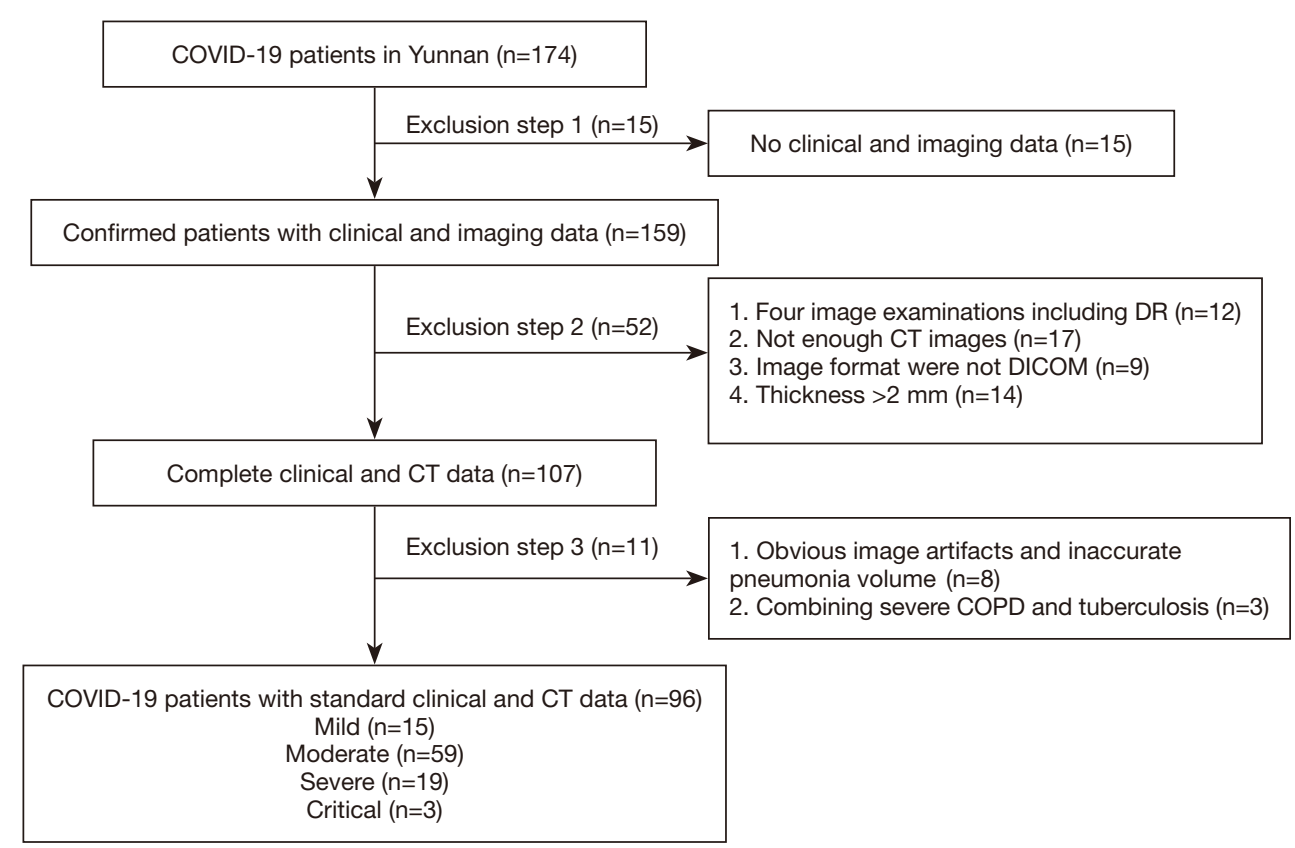

Figure 1 Flow diagram of the study design and participants included in the analysis.

liver disease, and tumors), date of CT scan, and dates of admission and discharge. Based on disease severity, the COVID-19 patients were classified into four types: mild, moderate, severe, and critical (2). All patients were from Yunnan Province, China, and received treatment according to the treatment guidelines in the hospital.

\section{CT protocol}

Spiral CT scanners were used with 16 or more detector rows (Philips, Netherlands; GE, United States; Siemens, Germany; or United Imaging, China). Patients were placed in the supine position and instructed to hold their breath during scanning, which occurred from the thoracic inlet to the costophrenic angle. The scanning parameters were as follows: detector collimation width, $64 \times 0.6$ or $128 \times$ $0.6 \mathrm{~mm}$; tube voltage, $120 \mathrm{kV}$; adaptive tube current; and high-resolution algorithm reconstruction (thickness: 1 or $1.5 \mathrm{~mm}$, interval $1.5 \mathrm{~mm}$ ).

\section{Chest CT evaluation}

Three Chinese radiologists who were blinded to the RTPCR results, all patient information and COVID-19 severity. Two experienced radiologists in the Department of Cardiothoracic Radiology (YH and YJ) independently read the CT images and then discussed any discrepancies (if any), which were further reviewed by a senior radiologist in the Department of Cardiothoracic Radiology (BH). The $\mathrm{CT}$ findings, CT value, and lesion volume were analyzed for stage 1 (days $0-4$ ), stage 2 (days 5-9), stage 3 (days 10-14), and stage 4 (days 15-19). CT findings (9) included lesion distribution (peripheral lung field, central lung field, or none), location (left upper lobe, left lower lobe, right upper lobe, right middle lobe, and right lower lobe), and the number and features of lesions (round GGOs, partial consolidation, consolidation, fibrous stripes, subpleural lines, and bronchiectasis). The CT value and lesion volume were measured at a window width/window level of 1,600/-600 HU. The lesions were manually delineated at a postprocessing workstation (Intelli Space Portal v6.0.1.20500, Philips, Netherlands) to obtain the mean CT value. The software automatically calculated the total lung volume and identified the lesions. After manual vessel removal, the volume of each lesion was measured, and then the total lesion volume was calculated (Figure 2).

\section{Statistical analysis}

SAS v9.4 (SAS Institute, Inc., SAS, Cary, NC, USA) was used for the statistical analyses. Count data were expressed as frequency, and measurement data were expressed as $\bar{x} \pm \mathrm{s}$. 

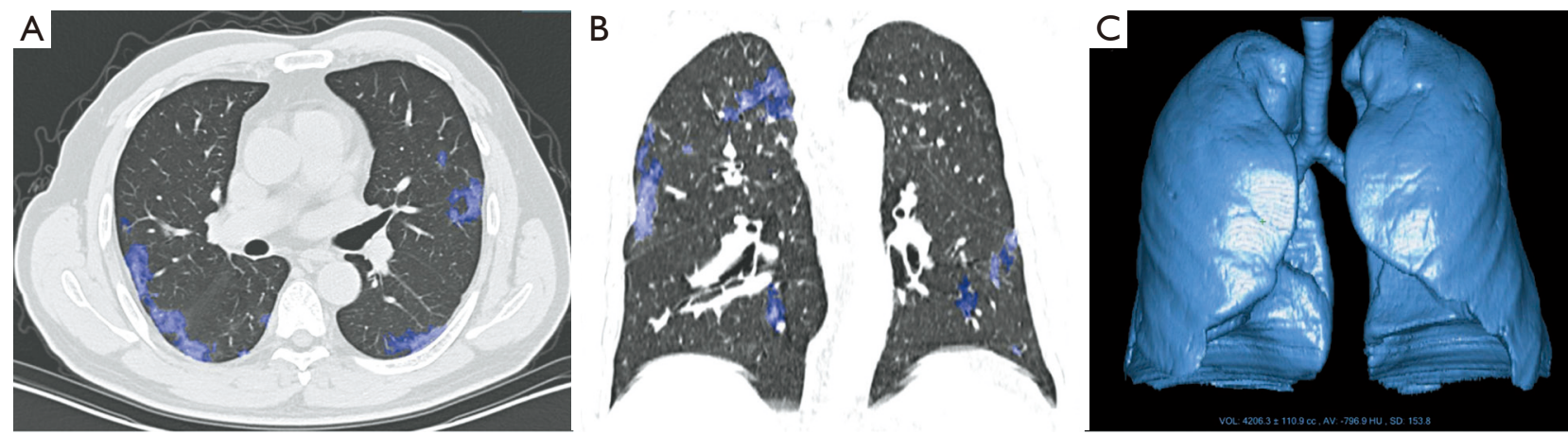

Figure 2 The process of measuring pneumonia volume based on HRCT. (A) Axial HRCT image of pneumonia (pneumonia lesions are marked blue); (B) coronal HRCT image of pneumonia (pneumonia lesions are marked blue); (C) total lung volume. HRCT, high-resolution computed tomography.

The distribution, location, number, and type of lesions over the four disease stages were analyzed with the $\chi^{2}$ test or Fisher's exact test. The CT value, lesion volume, and lesion volume percentage were first analyzed for normal distribution. Normally distributed data were analyzed with one-way analysis of variance (ANOVA), and the least significant difference (LSD) and Tamhane's test was used for data conforming or not conforming to homogeneity of variance for pairwise comparisons, respectively. Nonnormally distributed data were analyzed with the Kruskal-Wallis rank sum test, and the Mann-Whitney test was used for pairwise comparisons. $\mathrm{P}<0.05$ was considered statistically significant.

\section{Results}

\section{Patient characteristics}

A total of 96 patients, including 46 men and 50 women aged $43.40 \pm 17.62$, were included in this study. All patients had a history of traveling or residing in Hubei or having close contact with COVID-19 patients. The most common clinical symptoms were fever (48.96\%) and cough (43.75\%), while $10.42 \%$ of the patients showed no symptoms. The patients had no apparent underlying diseases. Based on disease severity, the patients were classified into four types: mild $(15,15.63 \%)$, moderate $(59,61.46 \%)$, severe (19, 19.79\%), and critical (3, 3.13\%). For all patients, the first lung CT was performed within 4 days of onset, the mean CT scan number was $4 \pm 1$, and the mean hospital stay was $15 \pm 4$ days (13-24 days). Table 1 shows the clinical characteristics of these patients.

\section{Lesion distribution and location across the COVID-19 disease stages}

The CT findings were negative across the four disease stages for patients with mild COVID-19 (Figure $3 A, B, C, D$ ). Table 2 shows the lesion distribution and location across the four disease stages in patients with moderate, severe, and critical COVID-19. The results showed that there were no lesions solely in the central lung field of patients with COVID-19. For patients with moderate COVID-19, more lesions were found in the peripheral lung field across the four stages than in the peripheral + central lung fields $(\mathrm{P}<0.05)$, and the number of negative patients in stage 4 was more than that in stages $1-3(\mathrm{P}<0.05)$. In COVID-19 patients, more lesions were found in the left lower lobe and the right lower lobe than in other lobes $(\mathrm{P}<0.05)$ across all four stages. The number of patients per involved lobe did not significantly change from stage 1 to stage $4(\mathrm{P}>0.05)$ for all COVID-19 patients. However, the number of lobes involved decreased significantly from stages $1-3$ to stage 4 in moderate and severe patients $(\mathrm{P}<0.05)$.

\section{Dynamic changes in CT findings across the disease stages of COVID-19 patients}

We compared the CT findings (six lesion features) among the total, moderate, severe, and critical COVID-19 patients across the disease stages (Figure 3E,F,G,H,I,7,K,L, Figures 4-6). Significant differences were observed in round GGOs, partial consolidation, fibrous stripes, and subpleural lines across the disease stages in all patients and in patients with moderate COVID-19 (Figure 3E,F,G,H,I,7,K,L, Figure 6A,B, 
Table 1 Basic clinical characteristics of 96 patients with COVID-19

\begin{tabular}{|c|c|}
\hline Clinical characteristics & All patients $(n=96)$ \\
\hline Age average & $43.40 \pm 17.62$ \\
\hline Age range & $5-79$ \\
\hline \multicolumn{2}{|l|}{ Gender } \\
\hline Male & $46(47.92 \%)$ \\
\hline Female & $50(52.08 \%)$ \\
\hline \multicolumn{2}{|l|}{ Epidemiological history } \\
\hline Travel/residence history in Hubei & $71(73.96 \%)$ \\
\hline $\begin{array}{l}\text { Close contact with confirmed } \\
\text { patients }\end{array}$ & 25 (26.04\%) \\
\hline \multicolumn{2}{|l|}{ Symptoms } \\
\hline Fever & 47 (48.96\%) \\
\hline Cough & $42(43.75 \%)$ \\
\hline Fatigue & $12(12.50 \%)$ \\
\hline Difficulty breathing & $2(2.08 \%)$ \\
\hline Sore throat & $11(11.46 \%)$ \\
\hline Runny nose & $8(8.33 \%)$ \\
\hline Expectorant & $16(16.67 \%)$ \\
\hline Headache & $4(4.17 \%)$ \\
\hline Muscle ache & 7 (7.29\%) \\
\hline Chest tightness & $7(7.29 \%)$ \\
\hline Chest pain & $2(2.08 \%)$ \\
\hline Vomiting & $2(3.08 \%)$ \\
\hline Diarrhea & $1(1.04 \%)$ \\
\hline No obvious symptoms & $10(10.42 \%)$ \\
\hline \multicolumn{2}{|l|}{ Basic disease } \\
\hline Hypertension & 7 (7.29\%) \\
\hline Diabetes & $6(6.25 \%)$ \\
\hline Coronary heart disease & $2(2.08 \%)$ \\
\hline Liver disease & $1(1.04 \%)$ \\
\hline Malignant tumor & $0(0 \%)$ \\
\hline \multicolumn{2}{|l|}{ Clinical typing } \\
\hline Mild & $15(15.63 \%)$ \\
\hline Moderate & $59(61.46 \%)$ \\
\hline Severe & 19 (19.79\%) \\
\hline Critical & 3 (3.13\%) \\
\hline
\end{tabular}

Continuous data are expressed as mean $\pm \mathrm{SD}$, and categorical data are presented as n (\%). COVID-19, coronavirus disease 2019. all $\mathrm{P}<0.05)$. Round GGOs decreased from stage 1 to stage 4; partial consolidation peaked in stage 2 and then decreased in stages 3-4; fibrous stripes and subpleural lines increased from stage 1 and peaked in stage 4. Significant differences were observed in partial consolidation, consolidation, fibrous stripes, and subpleural lines across the disease stages in patients with severe COVID-19 (Figure 4A,B,C,D,E,F, G, $H, I, \mathcal{F}, K, L, M, N, O, P$, Figure $6 C$, all $\mathrm{P}<0.05)$, but no significant difference was observed in round GGOs in patients with severe disease $(\mathrm{P}>0.05)$. Moreover, partial consolidation and consolidation were more common in patients with severe disease than in patients with moderate disease. Patients with critical disease showed significant extensive partial consolidation and consolidation. Compared with other types of COVID-19 patients, patients with critical illness showed bronchiectasis (Figure $5 A, B, C, D, E, F, G, H, I, \mathcal{F}, K, L, M$, N,O,P, Figure 6D).

\section{Lesion number, CT value, and lesion volume across the disease stages of COVID-19 patients}

We analyzed the lesion number, CT value, and lesion volume across the disease stages (Figure 7). No significant difference was observed in lesion number from stages 1-3 to stage 4 in all COVID-19 patients and, separately, in patients with moderate and severe COVID-19 (all P>0.05); however, the CT value, lesion volume, and lesion volume percentage significantly decreased from stages $1-2$ to stage 4 (Figure $7 A, B, C, E, F, G, I, \mathcal{F}, K, M, N, O$, all $\mathrm{P}<0.05$ ). In critical patients, no significant differences were observed in lesion number and lesion volume across the disease stages (Figure $7 D, L$, both $\mathrm{P}>0.05$ ); however, the $\mathrm{CT}$ value and lesion volume percentage decreased significantly across the disease stages (Figure $7 H, P$, both $\mathrm{P}<0.05$ ). Figure 8 summarizes the dynamic changes in CT findings of COVID-19 patients with different severities across the disease stages.

\section{Discussion}

COVID-19 is a viral infection that has recently spread worldwide. In this study, we systematically described the dynamic changes in chest CT findings of patients with different COVID-19 severities across the disease stages. The results showed that COVID-19-related lung lesions progressed rapidly and peaked at days 5-9 after admission. The CT findings varied in the COVID-19 patients with different severities, and the appearance and trends for different lesion types were different; however, each lesion 
Table 2 Distribution and location of lung lesions in the four stages

\begin{tabular}{|c|c|c|c|c|c|c|c|c|c|c|}
\hline Severity & Stages & No findings & Peripheral & $\begin{array}{c}\text { Peripheral + } \\
\text { central }\end{array}$ & $\begin{array}{l}\text { Upper left } \\
\text { lobe }\end{array}$ & $\begin{array}{l}\text { Left lower } \\
\text { lobe }\end{array}$ & $\begin{array}{c}\text { Right upper } \\
\text { lobe }\end{array}$ & $\begin{array}{l}\text { Right lung } \\
\text { middle lobe }\end{array}$ & $\begin{array}{c}\text { Right lower } \\
\text { lobe }\end{array}$ & $\begin{array}{c}\text { Number of } \\
\text { involved lobes }\end{array}$ \\
\hline \multirow[t]{3}{*}{ Moderate } & Stage 1 & 3 & $56^{\#}$ & 0 & 25 & 42 & 27 & 19 & 41 & 154 \\
\hline & Stage 3 & 6 & $53^{\#}$ & 0 & 25 & 40 & 25 & 15 & 45 & 150 \\
\hline & Stage 4 & 13 & $46^{\#}$ & 0 & 19 & 29 & 18 & 10 & 38 & $114^{*}$ \\
\hline \multirow{3}{*}{ Severe } & Stage 2 & 0 & 11 & 8 & 16 & 15 & 13 & 11 & 15 & 70 \\
\hline & Stage 3 & 0 & $14^{\#}$ & 5 & 12 & 15 & 12 & 10 & 12 & 61 \\
\hline & Stage 4 & 2 & $15^{\#}$ & 2 & 11 & 12 & 11 & 8 & 10 & $52^{*}$ \\
\hline Critical & Stage 1 & 0 & 1 & 2 & 3 & 3 & 3 & 3 & 3 & 15 \\
\hline
\end{tabular}

Data are number of patients. ", peripheral compared with no findings and peripheral + central, significance was considered when $\mathrm{P}<0.05$; *, stage 4 compared with stages 1-3, significance was considered when $\mathrm{P}<0.05$.

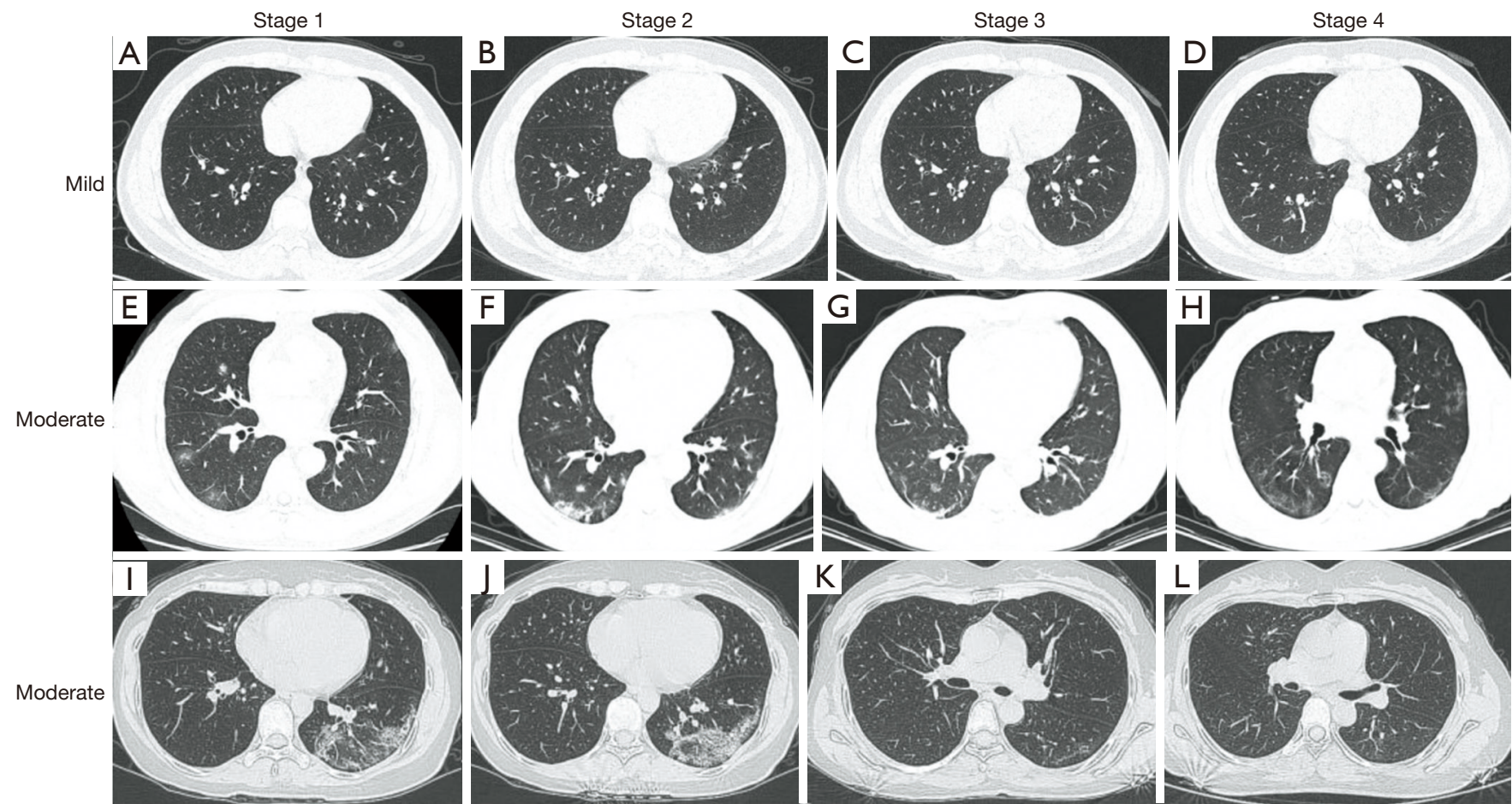

Figure 3 CT findings for lung lesions in patients with mild and moderate COVID-19 across disease stages. (A,B,C,D) Male, 19 years old, mild COVID-19, axial view by CT scan. CT findings were negative in all four clinical stages. (E,F,G,H) Male, 56 years old, moderate COVID-19, axial view by CT scan. Several round GGOs in the right lower lobe and the left upper lobe (E), increased lesion scope and lesion number, with partial consolidation formed inside lesions (F), and subpleural lines after substantial lesion absorption (G,H). (I,J,K,L) Female, 37 years old, moderate COVID-19, axial view by CT scan. Multiple GGOs in the left lower lobe with interlobular septal thickening (I) and patchy lesions. Partial consolidation after disease progression (J), substantial lesion absorption after treatment with the appearance of subpleural lines (K), and finally complete lesion absorption (L). COVID-19, coronavirus disease 2019; CT, computed tomography; GGOs, ground glass opacities. 


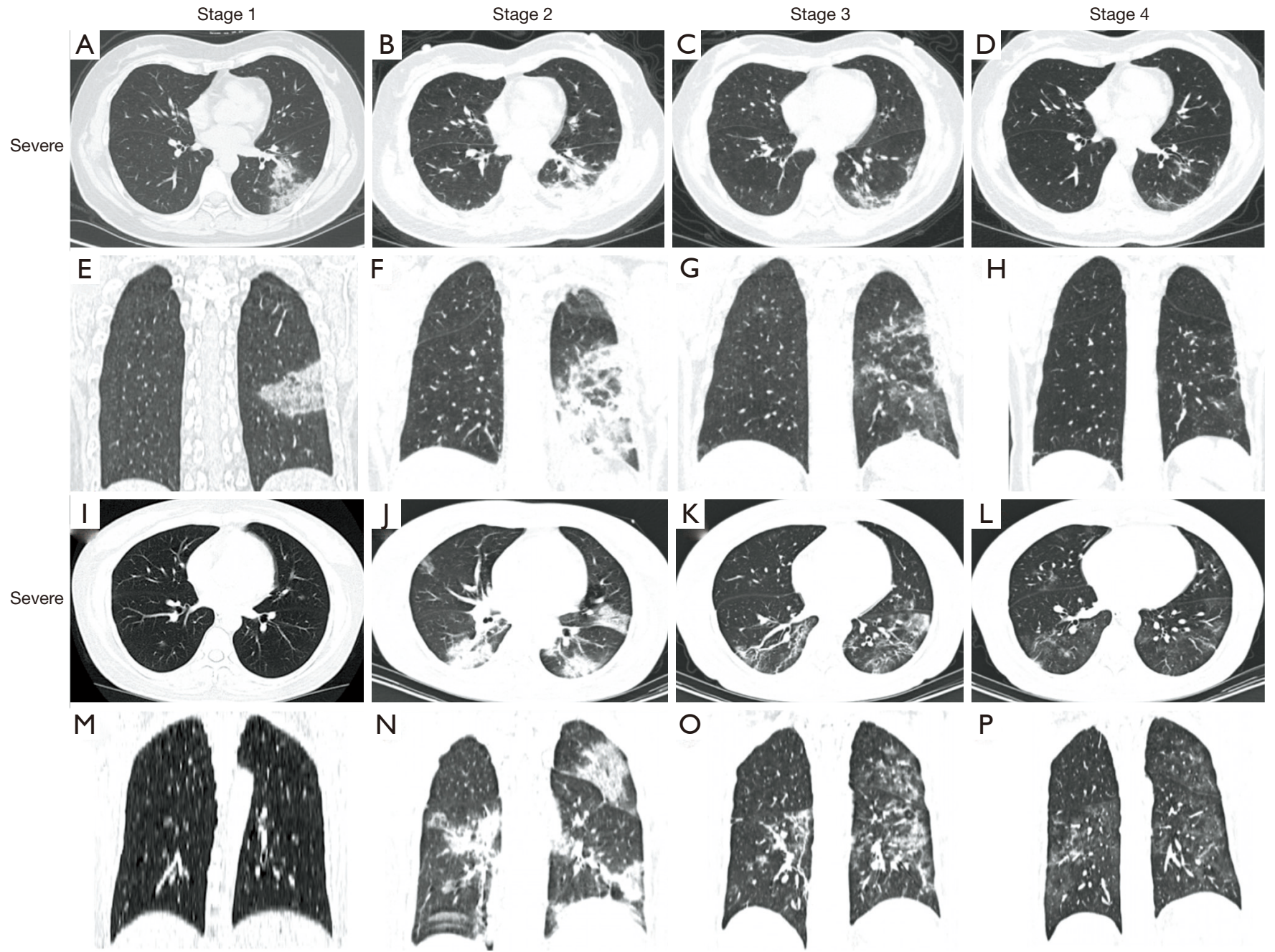

Figure 4 CT findings for lung lesions in patients with severe COVID-19 across disease stages. (A,B,C,D,E,F,G,H) Female, 49 years old, axial and coronal views by chest CT. Lesions in the left lower lobe (A,E), with significant consolidation in 5 days (B,F), followed by lesion absorption after treatment and residual subpleural lines (C,D,G,H). (I,J,K,L,M,N,O,P) Female, 42 years old, axial and coronal views by chest CT. Round GGOs in the right lower lobe and the left upper lobe (I,M), with rapid progression to consolidation and increased lesion density and lesion volume (J,N), followed by substantial lesion absorption after treatment and residual parenchymal bands (K,I,O,P). COVID-19, coronavirus disease 2019; CT, computed tomography; GGOs, ground glass opacities.

type exhibited a specific pattern.

All patients included in this study underwent four or more CT scans. One patient died during hospitalization. The most common clinical symptoms of COVID-19 patients in Yunnan were fever (48.96\%) and cough (43.75\%), results that are consistent with previous reports (10). However, the incidences of fever and cough were lower in this study, which may be related to the time between disease onset and admission. It should be noted that in this study, no activity and new lesions in CT examination across the disease stages in 10 asymptomatic patients (10.42\%) and 15 mild patients (15.63\%), results that are consistent with a study conducted by Guan et al. (11). In the study by Guan et al., the patients tested positive for SARS-CoV-2 by RTPCT and were infectious; therefore, isolation and antiviral therapy were required.

In this study, COVID-19 frequently affected the periphery of the lower lobes but rarely affected the right middle lobe, which was consistent with previous reports $(8,12)$. This may be because the bronchus in the lower lobe of the right lung is 


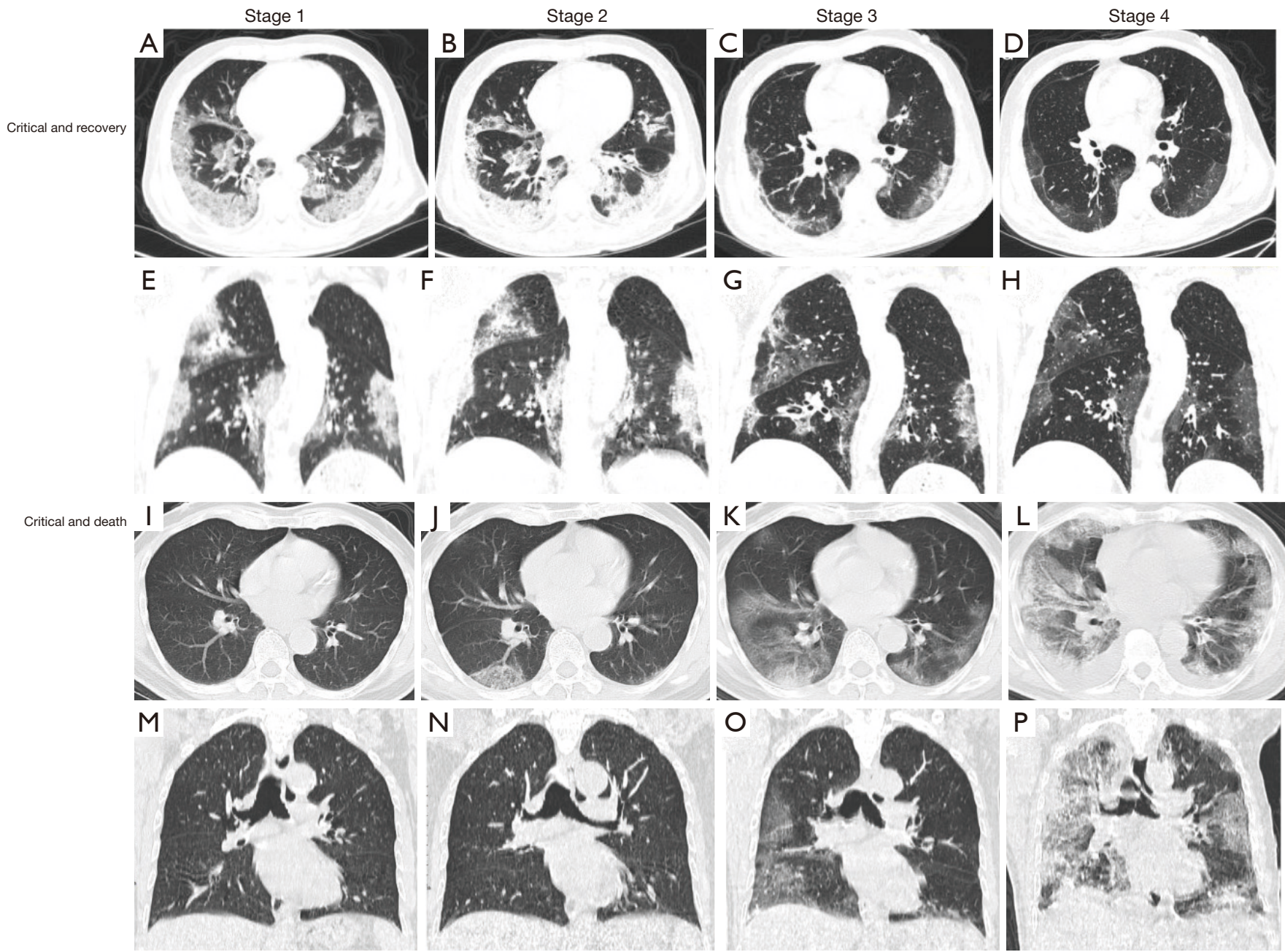

Figure 5 CT findings for lung lesions in patients with critical COVID-19 across disease stages. (A,B,C,D,E,F,G,H) Male, 58 years old, axial and coronal views by chest CT. Bilateral lesions and bronchiectasis (A,E), with rapid progression and significant consolidation (B,F), followed by substantial lesion absorption after 7 days of treatment and apparent subpleural lines (C,D,G,H). (I,J,K,L,M,N,O,P) Male, 72 years old, axial and coronal views by chest CT. Admission after fever for 2 days, lesion expansion in the lungs during hospitalization, white lung on day 17 of admission, and death 2 days later (I,P). COVID-19, coronavirus disease 2019.

thicker and shorter, and study have also shown that SARS$\mathrm{CoV}-2$ target cells may be located in the lower respiratory tract (13). For patients with extremely severe disease, the lesions extended to other lobes and the central lung field, which in turn was a sign of disease progression and exacerbation. This was consistent with the lesion distribution of patients with different COVID-19 severities reported by Liu et al. (14).

This study showed that round GGOs and partial consolidation were the most common CT findings for patients with moderate and severe COVID-19. In the early stage (days 0-4), round GGOs and partial consolidation were observed in $96.30 \%$ and $45.57 \%$ of the patients, respectively. As the disease progressed, round GGOs decreased over time, while some round GGOs progressed to partial consolidation $(15,16)$, which peaked on days 5-9. On days $10-15$, fibrous stripes and subpleural lines increased, suggesting substantial lesion absorption $(17,18)$. CT showed complete lesion absorption in 15 patients at discharge. In critical patients, lesions were more widespread, CT findings were more diverse, and absorption after treatment was slower compared to that in other patients (19). Our results show that the progression of chest CT findings is closely related to the prognosis of COVID-19. With the decrease of pure GGO, the increase of consolidation, the expansion 

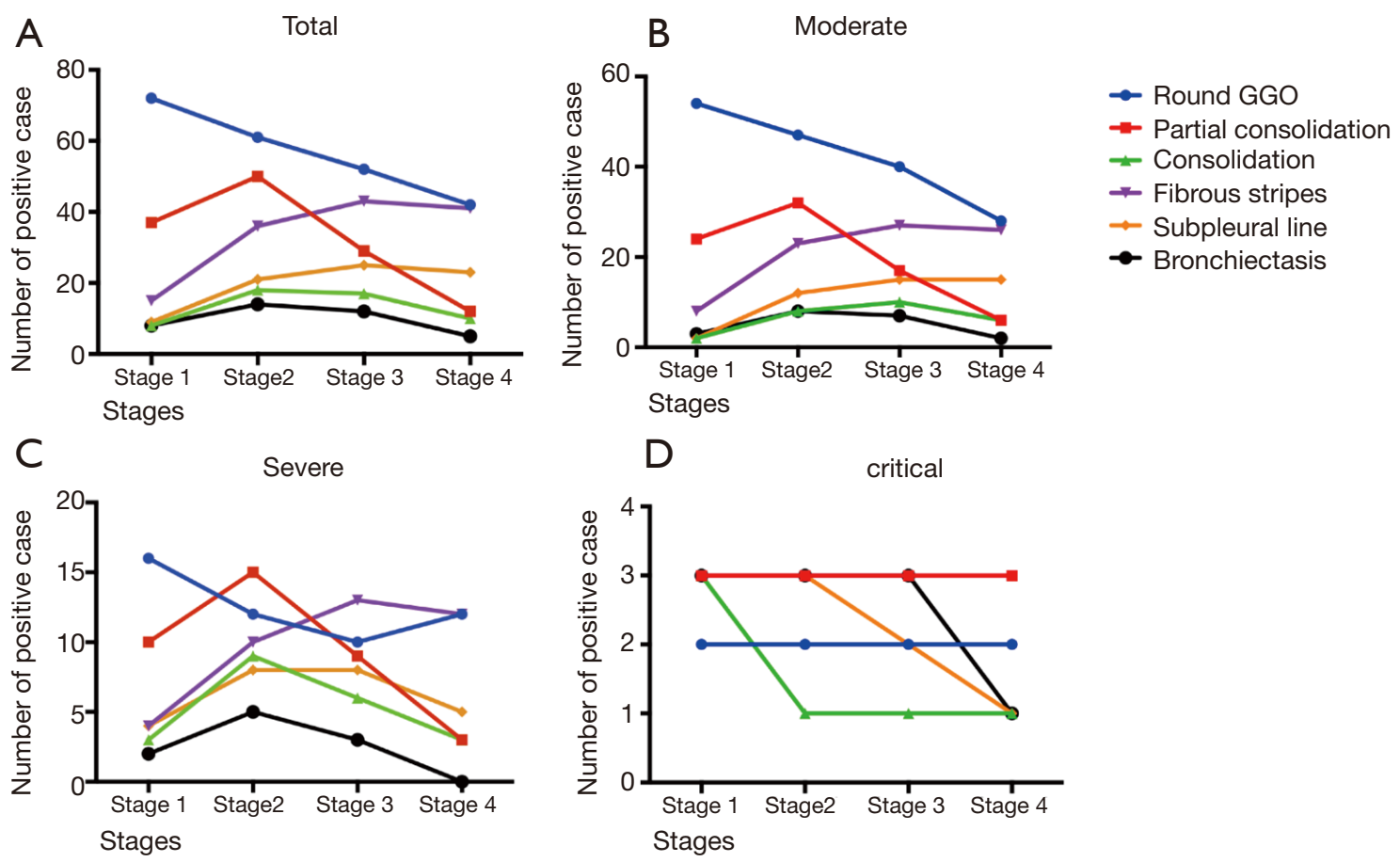

Figure 6 Dynamic changes in CT features across the four stages of COVID-19. (A) Total values for six CT features for all three disease severities; (B) moderate; (C) severe; and (D) critical. COVID-19, coronavirus disease 2019; CT, computed tomography; GGOs, ground glass opacities.

of the lesion area, and the appearance of crazy-paving pattern, the COVID-19 patient's prognosis becomes worse. Diagnosing COVID-19 in the early stage is also beneficial to improve the prognosis. If "white lung" is presented on CT images, the patient is at a high risk of a poor prognosis or even death (19-21).

Our results showed that the lesion number remained largely unchanged across the disease stages (especially for critical patients) but that the CT value and lesion volume significantly decreased over time, indicating that during the disease course, lung lesions were significantly absorbed, even though the lesion number remained unchanged. In critical patients, the total lung volume did not decrease significantly after treatment, but the lesion volume and lesion volume percentage decreased. At present, there are few studies analyzing the lesion density and lesion volume of COVID-19 patients, and our results showed that the dynamic CT value and lesion volume can provide a basis for the objective quantitative assessment of lesion absorption or exacerbation. Lesion absorption was not yet complete in $68.75 \%$ of the patients at discharge; however, viral nucleic acid tests were negative. Most COVID-19 chest CT examination results and clinical manifestations are synchronized, but not all patients. Guan et al. found that $23.87 \%$ of critical COVID-19 patients and $5.20 \%$ of non-critical patients had no obvious abnormalities in chest CT (11). And one study has reported that four patients tested positive again for SARS-CoV-2 by RT-PCR at 5-13 days after discharge (22). CT examinations were necessary for patients who have tested positive for SARS-COV-2 again. CT can help determine whether there were new lesions in the lungs and assess the severity of lung lesions. Therefore, it is important that patients continue to stay at home and be followed-up after discharge.

This study has some limitations. First, while we collected the clinical and imaging data of all COVID-19 patients in Yunnan Province, only 96 patients were included in the study. Second, because all patients were diagnosed early and were under active treatment, only three patients in this study progressed to a critical condition. Third, CT findings may vary with age groups, such as infants, children, or the elderly. We were unable to perform subgroup analysis by age group due to the small sample size. In the future, we may investigate dynamic CT findings in different 

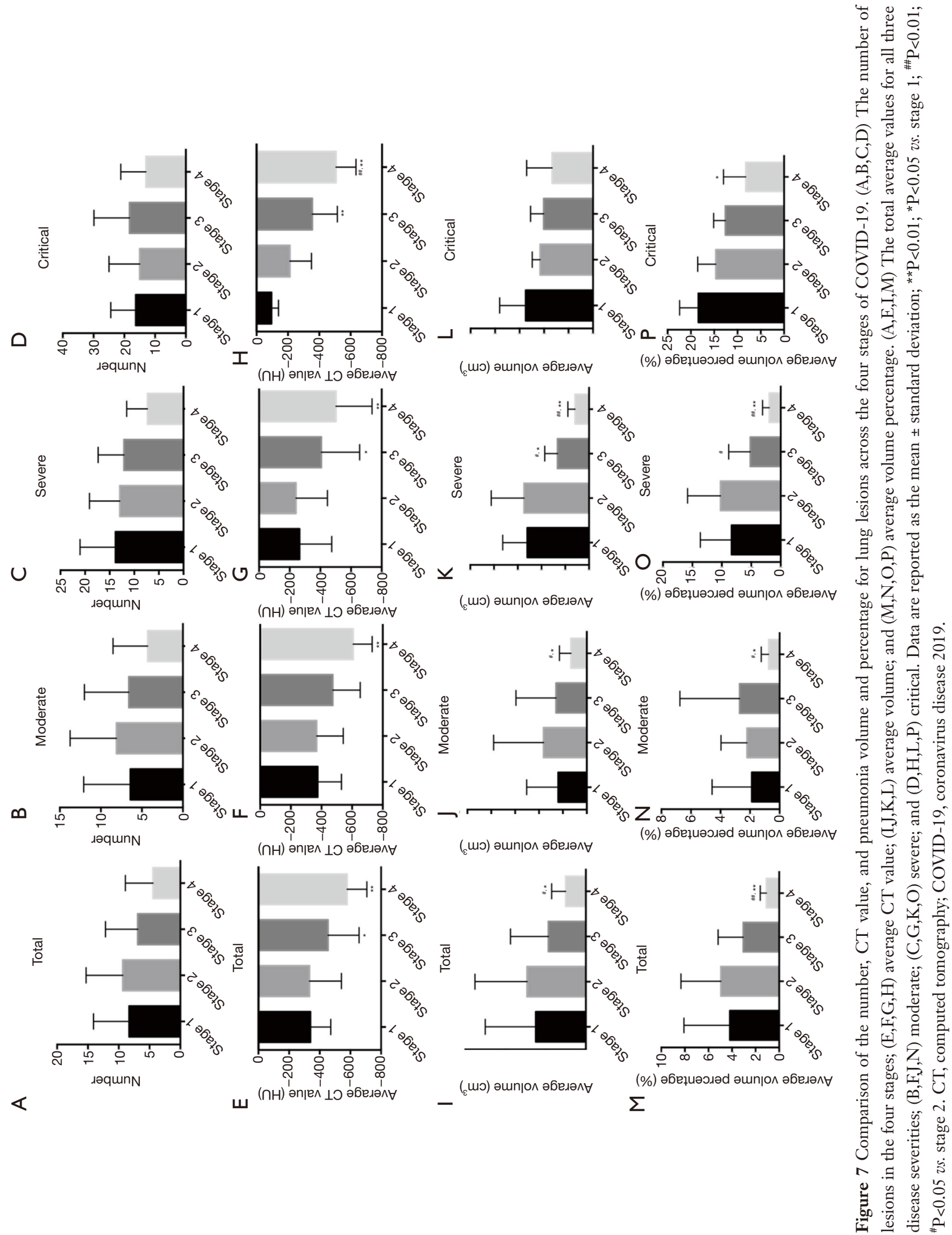


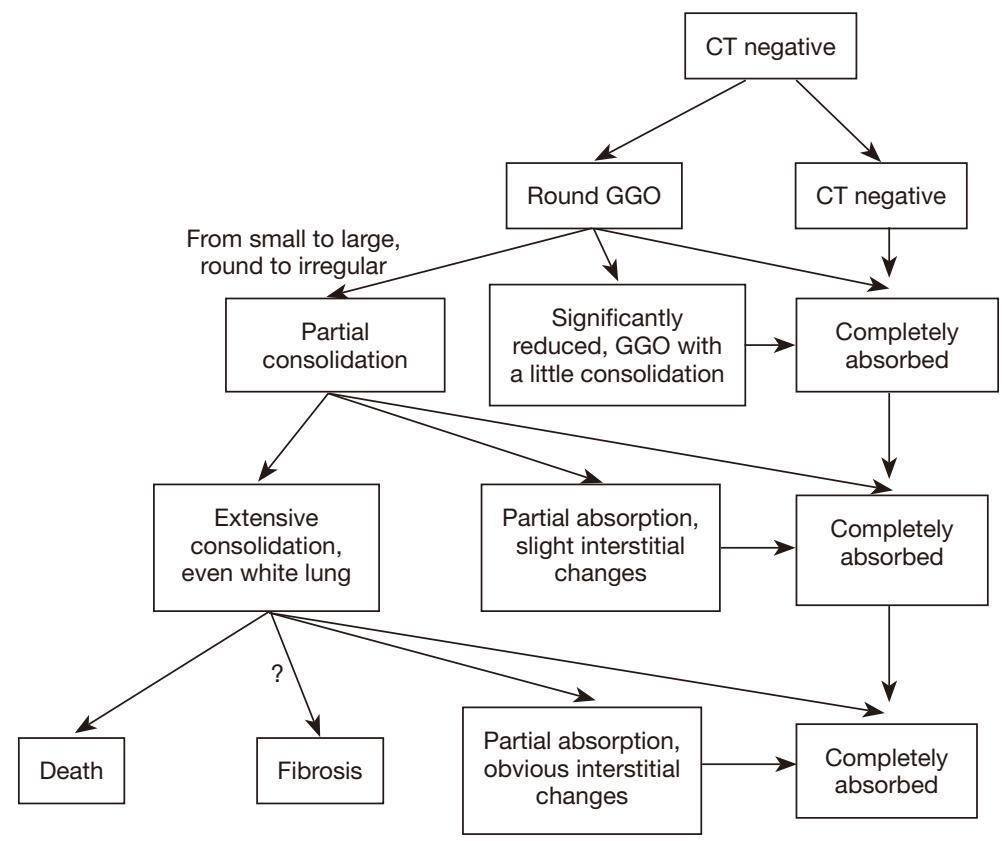

Figure 8 Evolution of lung lesions in COVID-19 patients. CT, computed tomography; GGOs, ground glass opacities; COVID-19, coronavirus disease 2019.

populations.

In summary, we investigated the CT findings of patients with different COVID-19 severities across the disease stages and confirmed that dynamic changes in chest CT findings were of important clinical value in evaluating the evolution of pulmonary lesions in COVID-19 patients. These results are helpful for the early diagnosis and assessment of the development of COVID-19 and for formulating the appropriate clinical treatment strategies.

\section{Acknowledgments}

The authors would like to express their appreciation to all hospital staff for their efforts in combating the COVID-19 outbreak. Thanks to all the COVID-19 patients who participated in this study.

Funding: None.

\section{Footnote}

Reporting Checklist: The authors have completed the MDAR checklist. Available at http://dx.doi.org/10.21037/ apm-20-2484

Data Sharing Statement: Available at http://dx.doi. org/10.21037/apm-20-2484

Conflicts of Interest: All authors have completed the ICMJE uniform disclosure form (available at http://dx.doi. org/10.21037/apm-20-2484). The authors have no conflicts of interest to declare.

Ethical Statement: The authors are accountable for all aspects of the work in ensuring that questions related to the accuracy or integrity of any part of the work are appropriately investigated and resolved. The study was conducted in accordance with the Declaration of Helsinki (as revised in 2013). The retrospective study was approved by the institutional committee of the First Affiliated Hospital of Kunming Medical University. Informed consent was waived because the study was retrospective in design.

Open Access Statement: This is an Open Access article distributed in accordance with the Creative Commons Attribution-NonCommercial-NoDerivs 4.0 International License (CC BY-NC-ND 4.0), which permits the noncommercial replication and distribution of the article with the strict proviso that no changes or edits are made and the original work is properly cited (including links to both the formal publication through the relevant DOI and the license). 
See: https://creativecommons.org/licenses/by-nc-nd/4.0/.

\section{References}

1. Zhu N, Zhang D, Wang W, et al. A Novel Coronavirus from Patients with Pneumonia in China, 2019. N Engl J Med 2020;382:727-33.

2. China National Health Commission. Diagnosis and treatment of pneumonitis caused by new coronavirus (trial version 6). Available online: http://www.nhe.gov.en/yzygj/ s7653p/202002/8334a8326dd94d329df351d7da8aefe2. shtml. Accessed February 19, 2020.

3. Ai T, Yang Z, Hou H, et al. Correlation of Chest CT and RT-PCR Testing for Coronavirus Disease 2019 (COVID-19) in China: A Report of 1014 Cases. Radiology 2020;296:E32-E40.

4. $\mathrm{Hu} \mathrm{Z}$, Song $\mathrm{C}, \mathrm{Xu} \mathrm{C}$, et al. Clinical characteristics of 24 asymptomatic infections with COVID-19 screened among close contacts in Nanjing, China. Sci China Life Sci 2020;63:706-11.

5. Ling Z, Xu X, Gan Q, et al. Asymptomatic SARS-CoV-2 infected patients with persistent negative CT findings. Eur J Radiol 2020;126:108956.

6. Weiss P, Murdoch DR. Clinical course and mortality risk of severe COVID-19. Lancet 2020;395:1014-5.

7. Matthay MA, Aldrich JM, Gotts JE. Treatment for severe acute respiratory distress syndrome from COVID-19. Lancet Respir Med 2020;8:433-4.

8. Shi H, Han X, Jiang N, et al. Radiological findings from 81 patients with COVID-19 pneumonia in Wuhan, China: a descriptive study. Lancet Infect Dis 2020;20:425-34.

9. $\mathrm{Xu} \mathrm{X}, \mathrm{Yu} \mathrm{C}, \mathrm{Qu} \mathrm{J}$, et al. Imaging and clinical features of patients with 2019 novel coronavirus SARS-CoV-2. Eur J Nucl Med Mol Imaging 2020;47:1275-80.

10. Wang D, Hu B, Hu C, et al. Clinical Characteristics of 138 Hospitalized Patients With 2019 Novel CoronavirusInfected Pneumonia in Wuhan, China. JAMA 2020;323:1061-9.

11. Guan WJ, Ni ZY, Hu Y, et al. Clinical Characteristics of Coronavirus Disease 2019 in China. N Engl J Med 2020;382:1708-20.

12. Bai HX, Hsieh B, Xiong Z, et al. Performance of Radiologists in Differentiating COVID-19 from NonCOVID-19 Viral Pneumonia at Chest CT. Radiology 2020;296:E46-E54.

13. Phan LT, Nguyen TV, Luong QC, et al. Importation and
Human-to-Human Transmission of a Novel Coronavirus in Vietnam. N Engl J Med 2020;382:872-4.

14. Liu KC, Xu P, Lv WF, et al. CT manifestations of coronavirus disease-2019: A retrospective analysis of 73 cases by disease severity. Eur J Radiol 2020;126:108941.

15. Pan F, Ye T, Sun P, et al. Time Course of Lung Changes at Chest CT during Recovery from Coronavirus Disease 2019 (COVID-19). Radiology 2020;295:715-21.

16. Wang Y, Dong C, Hu Y, et al. Temporal Changes of CT Findings in 90 Patients with COVID-19 Pneumonia: A Longitudinal Study. Radiology 2020;296:E55-E64.

17. Xu YH, Dong JH, An WM, et al. Clinical and computed tomographic imaging features of novel coronavirus pneumonia caused by SARS-CoV-2. J Infect 2020;80:394-400.

18. Pan $Y$, Guan H, Zhou S, et al. Initial CT findings and temporal changes in patients with the novel coronavirus pneumonia (2019-nCoV): a study of 63 patients in Wuhan, China. Eur Radiol 2020;30:3306-9.

19. Li K, Wu J, Wu F, et al. The Clinical and Chest CT Features Associated With Severe and Critical COVID-19 Pneumonia. Invest Radiol 2020;55:327-31.

20. 2019. Xiong Y, Sun D, Liu Y, et al. Clinical and HighResolution CT Features of the COVID-19 Infection: Comparison of the Initial and Follow-up Changes. Invest Radiol 2020;55:332-9.

21. Wu C, Chen X, Cai Y, et al. Risk Factors Associated With Acute Respiratory Distress Syndrome and Death in Patients With Coronavirus Disease 2019 Pneumonia in Wuhan, China. JAMA Intern Med 2020;180:934-43.

22. Lan L, Xu D, Ye G, et al. Positive RT-PCR Test Results in Patients Recovered From COVID-19. JAMA 2020;323:1502-3.

(English Language Editor: D. Fitzgerald)

Cite this article as: Huang $\mathrm{Y}, \mathrm{Li} Z$, Guo H, Han D, Yuan F, Xie Y, Li Z, Zhang J, Wang P, Yang Y, Li Y, Ma J, Hu C, Wang $\mathrm{H}$, Zhao W, Yi W, Peng Y, Jiang Y, Li Z, Yang D, Wu K, Li Y, Feng Z, Liu C, Zhu G, Han Y, He S, He B, Zhang Z, Pi J. Dynamic changes in chest CT findings of patients with coronavirus disease 2019 (COVID-19) in different disease stages: a multicenter study. Ann Palliat Med 2021;10(1):572583. doi: 10.21037/apm-20-2484 